\title{
COMPORTAMENTO SORTIVO, INDIVIDUAL E COMPETITIVO, DE METAIS PESADOS EM LATOSSOLOS COM MINERALOGIA CONTRASTANTE ${ }^{(1)}$
}

\author{
Maria Aparecida Pereira Pierangeli ${ }^{(2)}$, Luiz Roberto Guimarães Guilherme ${ }^{(3)}$, \\ Nilton Curi ${ }^{(3)}$, Enio Tarso de Souza Costa ${ }^{(4)}$, José Maria de Lima ${ }^{(3)}$, João José \\ Granate de Sá e Melo Marques ${ }^{(3)} \&$ Luiz Framarion P. Figueiredo ${ }^{(5)}$
}

\section{RESUMO}

O efeito poluente de elementos-traço no ambiente depende, principalmente, da capacidade dos colóides do solo em sorvê-los e mantê-los retidos com alta energia. Neste trabalho, avaliou-se a adsorção e dessorção de $\mathrm{Cd}, \mathrm{Cu}$ e $\mathrm{Pb} \mathrm{em}$ amostras do horizonte A de Latossolo Vermelho e Latossolo Vermelho-Amarelo com textura semelhante e mineralogia contrastante. Para isso, foram construídas isotermas de adsorção desses três metais, individual e simultaneamente, por meio da reação das amostras de solo suspensas em $\mathrm{Ca}\left(\mathrm{NO}_{3}\right)_{2} 5 \mathrm{mmol} \mathrm{L} \mathrm{L}^{-1}(\mathrm{pH}$ ajustado para 5,5$)$, com soluções dos metais nas concentrações de $0 ; 0,01 ; 0,05 ; 0,10 ; 0,15 ;$ e $0,75 \mathrm{mmol} \mathrm{L}^{-1}$. Posteriormente, procedeu-se aos ensaios de dessorção dos elementos previamente adsorvidos em solução de $\mathrm{Ca}\left(\mathrm{NO}_{3}\right)_{2} 5 \mathrm{mmol} \mathrm{L}^{-1}$. $\mathrm{O}$ modelo de isoterma de Langmuir $(P \leq 0,01)$ se ajustou aos dados obtidos e ambos os solos apresentaram maior capacidade máxima de adsorção para $\mathrm{Cu}$ e $\mathrm{Pb}$, comparados ao $\mathrm{Cd}$. A adsorção simultânea reduziu a adsorção dos três metais, sendo as maiores reduções observadas para o $\mathrm{Cd}$. Houve baixa dessorção dos metais adsorvidos, indicando a ocorrência de histerese. O menor poder competitivo de $\mathrm{Cd}$, evidenciado pela redução da sua capacidade máxima de adsorção na presença dos outros metais, torna esse elemento altamente preocupante em solos tropicais mais intemperizados, como os Latossolos.

Temos de indexação: elementos-traço, poluição do solo, adsorção, dessorção.

\footnotetext{
(1) Recebido para publicação em abril de 2005 e aprovado em março de 2007.

(2) Universidade do Estado de Mato Grosso - UNEMAT - Pontes e Lacerda, Caixa Postal 92, CEP 78250-000, MT. E-mail: mapp2@terra.com.br

(3) Universidade Federal de Lavras - UFLA, Departamento de Ciência do Solo, Caixa Postal 3037, CEP 37200-000. Bolsista do CNPq. E-mails: guilherm@ufla.br; niltcuri@ufla.br; jmarques@ufla.br; jmlima@ufla.br

(4) Doutorando - Departamento de Ciência do Solo/UFLA. Bolsista da FAPEMIG. E-mail: eniotarso@yahoo.com.br

(5) Engenheiro-Agrônomo. E-mail: luisframarion@ufla.br
} 


\title{
SUMMARY: INDIVIDUAL AND COMPETITIVE SORPTION OF HEAVY METALS IN OXISOLS WITH CONTRASTING MINERALOGY
}

\begin{abstract}
The pollution effect of trace elements in the environment depends mainly on the capacity of soil colloids to sorb and maintain them in high energy bonds. This study evaluated the adsorption of $\mathrm{Cd}, \mathrm{Cu}$, and $\mathrm{Pb}$ in A-horizon samples of Red Latosol and RedYellow Latosol (Udox), with similar texture and contrasting mineralogy. For this purpose, isotherms of adsorption, monoelement and multielement were built, based on the soil sample reaction in $5 \mathrm{mmol} \mathrm{L} \mathrm{L}^{-1} \mathrm{Ca}\left(\mathrm{NO}_{3}\right)_{2}$ solution adjusted to $\mathrm{pH} 5.5$, with metal concentrations of $0 ; 0.01 ; 0.05 ; 0.10 ; 0.15$; and $0.75 \mathrm{mmol} \mathrm{L}^{-1}$. Desorption was then analyzed in $5 \mathrm{mmol} \mathrm{L} \mathrm{L}^{-1} \mathrm{Ca}\left(\mathrm{NO}_{3}\right)_{2}$ solution. The isotherms were adjusted to the Langmuir model $(P \leq 0.01)$. The maximum adsorption capacity of $\mathrm{Cu}$ and $\mathrm{Pb}$ was higher than of $\mathrm{Cd}$ in both soils. The simultaneous adsorption reduced the adsorption maximum capacity of the three metals. $\mathrm{Cd}$ was most reduced, indicating the lower competitive power than $\mathrm{Cu}$ and $\mathrm{Pb}$. There was low reversibility of the adsorbed metals, indicating occurrence of hysteresis. The low competitive power of $C d$, as indicated by the reduced maximum adsorption capacity in the presence of the other two metals, is alarming in weathered tropical soils, such as Oxisols.
\end{abstract}

Index terms: trace elements, soil pollution, adsorption, desorption.

\section{INTRODUÇÃO}

$\mathrm{O}$ aumento das concentrações de $\mathrm{Cd}, \mathrm{Pb}$ e $\mathrm{Cu}$ no ambiente pode ser devido a atividades industriais, deposição de resíduos contaminados e insumos agrícolas, entre outros (Kabata-Pendias \& Pendias, 2001). No Brasil, altas concentrações desses elementos têm sido relatadas, principalmente em áreas de depósito de resíduos de mineração (Ribeiro-Filho et al., 1999). No entanto, o uso de resíduos industriais como os estudados por Amaral Sobrinho et al. (1992) e Lima (2001) - e pesticidas (Ramalho et al., 2000), em áreas de produção agrícola, pode elevar substancialmente a concentração de elementos-traço nos solos, com conseqüentes efeitos adversos para a biota e os aqüíferos. Descrição dos efeitos deletérios nos homens devido a elevadas concentrações de elementos-traço em solos pode ser encontrada em Abrahams (2002).

A concentração dos elementos-traço na solução do solo e, conseqüentemente, sua biodisponibilidade e mobilidade são principalmente controladas por reações de adsorção e dessorção na superfície dos colóides do solo (Kabata-Pendias \& Pendias, 2001). De acordo com Strawn \& Sparks (2000), uma das principais causas de erros relacionados à previsão da potencial toxicidade de elementos-traço no solo é a falta de estudos sobre a dessorção destes nos sistemas de interesse.

Embora tanto a adsorção específica quanto a nãoespecífica ocorram em solos, a liberação de elementostraço adsorvidos especificamente tende a ser lenta ou incompleta (ocorrência de histerese), pois complexos de esfera interna requerem grande energia de ativação para o processo de dessorção (McBride, 1989). Tanto elevadas quanto pequenas proporções de metais dessorvidos por solos ou seus constituintes são relatadas na literatura por Backes et al. (1995), McLaren et al. (1998), Strawn \& Sparks (2000) e Weerasooriya et al. (2002). Grande disparidade das proporções de metais dessorvidos acontece em virtude da enorme diversidade de reagentes utilizados para promover a dessorção do elemento adsorvido: agentes complexantes (Lehmann \& Harter, 1984) ou soluções salinas (Backes et al., 1995). Para McLaren et al. (1998), soluções extratoras mais fracas são mais adequadas para simular a dessorção de metais pelos solos que os agentes complexantes.

O comportamento de elementos-traço nos solos tem sido avaliado mediante isotermas de adsorção e pode ser modelado por meio de equações matemáticas, como as equações de Langmuir, Freundlich, Toth e Redlich, entre outras (Pombo, 1995; Altin et al., 1998). A equação de Langmuir originalmente foi desenvolvida para a adsorção de um gás sobre uma superfície sólida, mas ela tem sido adaptada para reações entre constituintes do solo e da solução (Veith \& Sposito, 1977). Seus coeficientes consideram somente a ocorrência do fenômeno de adsorção; portanto, deve-se considerar na interpretação dos resultados que não somente a adsorção, mas também a precipitação, pode estar ocorrendo.

Normalmente, solos poluídos são simultaneamente contaminados por vários elementos, e a competição entre eles pode influenciar a sua mobilidade e biodisponibilidade (McBride, 1994; Temminghoff et al.,1995; Fontes et al., 2000). Além da competição, 
outros fatores - como concentração do metal, $\mathrm{pH}$, força iônica, CTC e os constituintes orgânicos e inorgânicos - também contribuem para a sua retenção no solo (Kabata-Pendias \& Pendias, 2001). A ação combinada desses fatores resulta em maior ou menor impacto ambiental devido à presença desses elementos nos solos.

Assim, neste trabalho, avaliou-se a adsorção e a dessorção de $\mathrm{Cd}$, $\mathrm{Cu}$ e $\mathrm{Pb}$, bem como o efeito competitivo entre esses metais, em amostras do horizonte A de dois Latossolos com textura semelhante e mineralogia contrastante, por meio de isotermas de adsorção mono e multielementares.

\section{MATERIAL E MÉTODOS}

Foram utilizadas amostras do horizonte $\mathrm{A}(0-20 \mathrm{~cm})$ de Latossolo Vermelho (LV) e Latossolo VermelhoAmarelo (LVA) com textura muito argilosa, teores intermediários de óxidos de Fe "totais" (165 $\mathrm{g} \mathrm{kg}^{-1}$ pelo ataque sulfúrico) e mineralogia contrastante. Esses solos foram selecionados em razão de sua mineralogia diferencial (mesmo se tratando de solos muitíssimos velhos), procurando-se isolar o efeito da granulometria e do teor "total" de óxidos de Fe que sabidamente interferem na reatividade desses sistemas. A zona fisiográfica escolhida para amostragem foi Campos das Vertentes - MG (município de São João Del Rei), que representa bem o bioma Cerrado, no qual os Latossolos são amplamente dominantes. No Quadro 1 sumarizam-se os principais atributos físicos, químicos e mineralógicos desses solos. Detalhes acerca dos métodos de determinação desses atributos podem ser encontrados em Lima \& Anderson (1997) e Guilherme \& Anderson (1998).

Para obtenção das isotermas de adsorção de $\mathrm{Cd}$, $\mathrm{Cu}$ e $\mathrm{Pb}$, transferiu-se 0,25 g de amostra de cada solo, em triplicata, para recipientes de $50 \mathrm{~mL}$, aos quais se adicionaram $16,75 \mathrm{~mL}$ de solução de $\mathrm{Ca}\left(\mathrm{NO}_{3}\right)_{2}$ $5 \mathrm{mmol} \mathrm{L}^{-1}$ (relação solo:solução 1:67), ajustando-se o $\mathrm{pH}$ da suspensão para 5,5 com solução saturada de
$\mathrm{Ca}(\mathrm{OH})_{2}$. Esse valor de $\mathrm{pH}$ foi escolhido por ser considerado bom para o desenvolvimento de plantas em condições tropicais e também por ser um valor em que a possibilidade de precipitação de metais catiônicos é limitada, seja na forma de óxidos, seja como carbonatos metálicos. Após atingido o $\mathrm{pH}$ de equilíbrio, foram adicionadas a cada suspensão, separadamente, soluções dos metais $\mathrm{Cd}, \mathrm{Cu}$ e $\mathrm{Pb}$, como sais nitrato, nas concentrações de $0 ; 0,03 ; 0,15 ; 0,30 ; 0,45$; e $2,25 \mathrm{mmol} \mathrm{L}^{-1}$ (relação solo:solução final 1:100; força iônica $15 \mathrm{mmol} \mathrm{L}^{-1}$; e concentrações iniciais (Ci) de metais para a adsorção $=0 ; 0,01 ; 0,05 ; 0,10 ; 0 ; 15$; e $\left.0,75 \mathrm{mmol} \mathrm{L}^{-1}\right)$. A fim de verificar o efeito competitivo dos três elementos, foram obtidas isotermas, nas mesmas condições anteriores, por meio da adição de soluções com, simultaneamente, os três elementos, nas mesmas concentrações descritas anteriormente. As suspensões foram agitadas por $72 \mathrm{~h}$ (alternandose $12 \mathrm{~h}$ de agitação e $12 \mathrm{~h}$ de repouso). Após esse período, as suspensões foram centrifugadas a $3.000 \mathrm{rpm}(1.600 \mathrm{~g})$ durante $20 \mathrm{~min}$ e o sobrenadante foi recolhido para determinação das concentrações dos metais na solução de equilíbrio (Ce), as quais foram determinadas por espectrofotometria de absorção atômica com chama ar-acetileno. A quantidade adsorvida foi calculada pela diferença entre a quantidade adicionada inicialmente $(\mathrm{Ci})$ e a Ce. A equação de Langmuir foi ajustada aos dados obtidos. Esta equação, em sua forma linear, pode ser representada como: $\mathrm{Ce} /(\mathrm{x} / \mathrm{m})=1 / \mathrm{Kb}+\mathrm{Ce} / \mathrm{b}$, em que Ce é a concentração do adsorbato na solução de equilíbrio; $\mathrm{K}$, a constante relacionada com a afinidade sorbato-adsorvente; $\mathrm{x} / \mathrm{m}$, a quantidade adsorvida por massa de adsorvente; e b, a capacidade máxima de adsorção. A linearização da isoterma pelo modelo de Langmuir foi realizada plotando-se, em um gráfico, $\mathrm{Ce} /(\mathrm{x} / \mathrm{m})$ contra Ce. Quando uma linha reta é ajustada a estes dados, o modelo de Langmuir é considerado adequado (Campbell \& Davies, 1995). De posse da equação da reta ajustada, os termos b (recíproco da inclinação) e K (recíproco do intercepto/b) foram obtidos. Determinou-se, assim, a capacidade máxima de adsorção dos metais (CMAM), para a adsorção de metais tanto individual quanto simultaneamente.

Quadro 1. Atributos físicos, químicos e mineralógicos do horizonte A dos Latossolos estudados

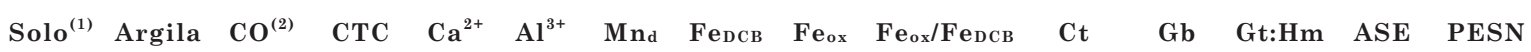

\begin{tabular}{|c|c|c|c|c|c|c|c|c|c|c|c|c|c|c|}
\hline LV & \multicolumn{2}{|c|}{$\mathrm{g} \mathrm{kg}^{-1}$} & \multicolumn{3}{|c|}{$\mathrm{cmol}_{\mathrm{c}} \mathrm{dm}^{-3}$} & \multicolumn{3}{|c|}{$\mathrm{g} \mathrm{kg}^{-1}$} & \multicolumn{3}{|c|}{$-\mathrm{g} \mathrm{kg}^{-1}$ argila - } & \multicolumn{3}{|c|}{$\mathrm{m}^{2} \mathrm{~g}^{-1}$} \\
\hline LVA & 711 & 15,8 & 5,1 & 0,4 & 0,2 & 0,30 & 101 & 1,20 & 0,012 & 480 & 375 & 10,2 & $54 \pm 3$ & 4,4 \\
\hline
\end{tabular}

(1) LV: Latossolo Vermelho; LVA: Latossolo Vermelho-Amarelo. ${ }^{(2)}$ CO: carbono orgânico; CTC: capacidade de troca catiônica a

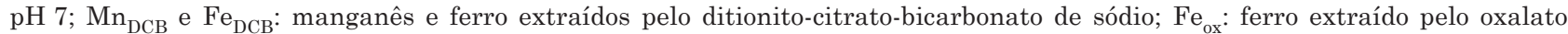
ácido de amônio; Ct: caulinita; Gb: gibbsita; Gt:Hm: relação goethita:hematita; ASE: área superficial específica; PESN: ponto de efeito salino nulo.

Fonte: Guilherme \& Anderson (1998); Lima \& Anderson (1997); Silva et al. (1996). 
Ao resíduo remanescente do experimento de adsorção nos tubos de centrífuga adicionaram-se $25 \mathrm{~mL}$ de $\mathrm{Ca}\left(\mathrm{NO}_{3}\right)_{2} 5 \mathrm{mmol} \mathrm{L}^{-1}$ (pH ajustado para 5,5), para determinação da dessorção dos metais $\mathrm{Cd}, \mathrm{Cu}$ e $\mathrm{Pb}$ retidos nas amostras de solo. Repetiu-se o mesmo procedimento com relação ao período de agitação e repouso realizado durante a adsorção. Após $72 \mathrm{~h}$, as amostras foram centrifugadas a $3.000 \mathrm{rpm}$ (1.600 g) durante $20 \mathrm{~min}$, e o sobrenadante, coletado para determinação das concentrações dos metais. A quantidade de metal dessorvido foi calculada, descontando-se aquela remanescente após a retirada do sobrenadante do experimento de adsorção. Os gráficos foram feitos utilizando-se o programa Sigma Plot (versão 9.0), sendo as médias e os desvios-padrão calculados de três repetições. A análise estatística foi feita utilizando-se o programa Sisvar, e as médias, submetidas ao teste de Scott-Knott a $5 \%$.

\section{RESULTADOS E DISCUSSÃO}

Nas menores concentrações iniciais (Ci), a maior parte do adicionado ao solo dos três metais foi adsorvida
(Figura 1), caracterizando forte afinidade dos seus constituintes para com $\mathrm{Cd}, \mathrm{Cu}$ e $\mathrm{Pb}$, principalmente quando estes estão em baixas concentrações no meio, corroborando prévios relatos (Strawn \& Sparks, 2000; Kabata-Pendias \& Pendias, 2001). Entretanto, à medida que a concentração inicial aumentou, menores quantidades, principalmente de $\mathrm{Cd}$ e $\mathrm{Pb}$, foram adsorvidas (Figura 1), indicando possível saturação dos sítios de adsorção. Em se tratando de retenção de elementos-traço por solos, é preciso ressaltar que existem outros mecanismos envolvidos, além da adsorção - complexação na superfície dos colóides, polimerização e fixação, por exemplo, são alguns desses mecanismos (Sparks, 1995; Bradl, 2004). Na retenção de Cd, Echeverría et al. (1998) relatam provável participação do mecanismo de complexação de superfície. Considerando-se que não somente a adsorção, mas também a precipitação, pode vir a constituir uma forma de redução dos elementos na concentração de equilíbrio, observam-se menores reduções nas quantidades adsorvidas para o $\mathrm{Pb}$ e o $\mathrm{Cu}$. Isso vem confirmar que a afinidade dos solos é maior para $\mathrm{Pb}$ e $\mathrm{Cu}$, conforme amplamente relatado na literatura (Fontes et al., 2000; Kabata-Pendias \&

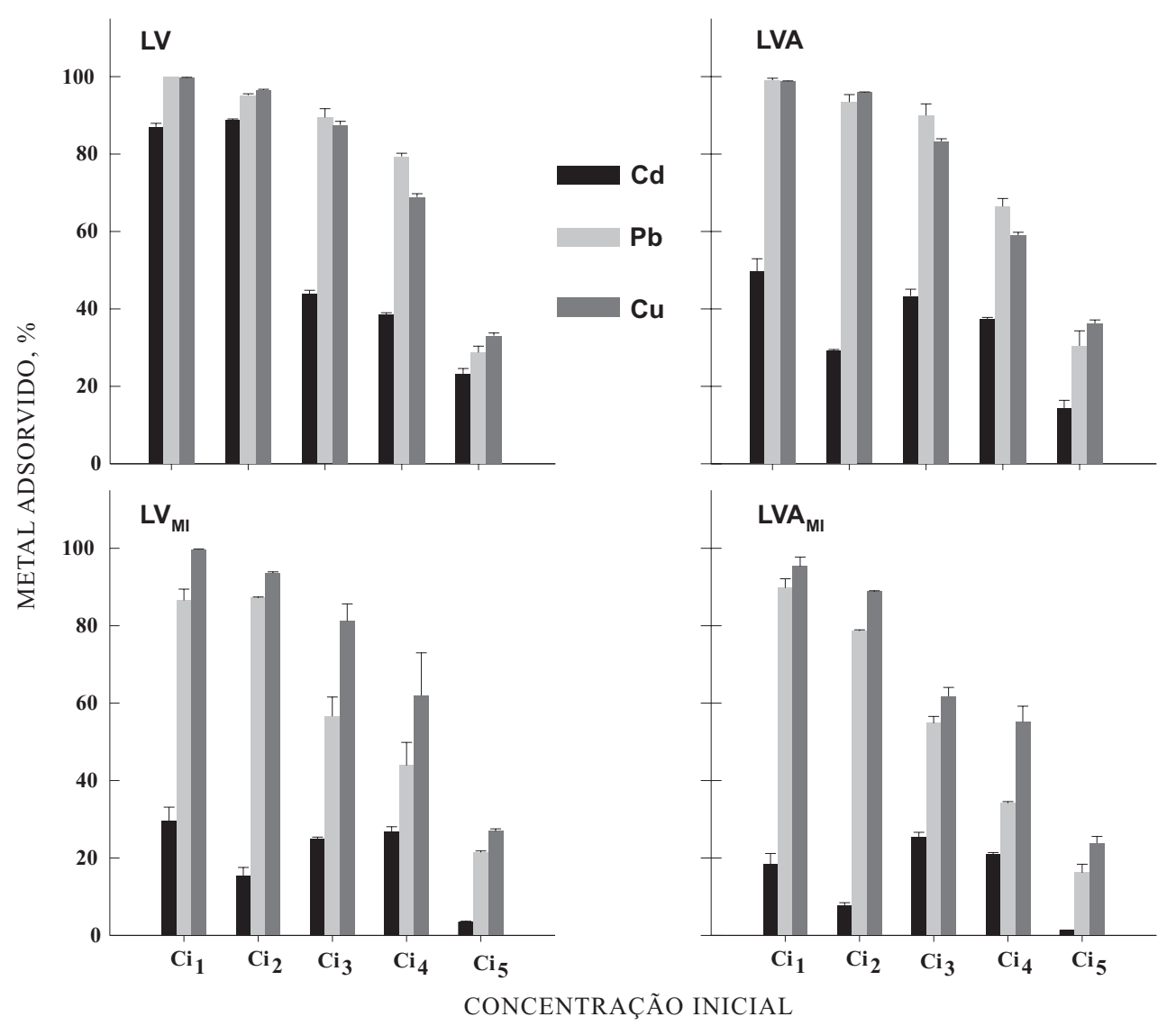

Figura 1. Porcentagem de $\mathrm{Cd}, \mathrm{Cu}$ e $\mathrm{Pb}$ adsorvidas por amostras de Latossolo Vermelho (LV) e Latossolo Vermelho-Amarelo (LVA), em função da quantidade de metal adicionada $\left(\mathrm{Ci}_{1}, \mathrm{Ci}_{2}, \mathrm{Ci}_{3}, \mathrm{Ci}_{4}\right.$ e $\mathrm{Ci}_{5}=0,01$; 0,$05 ; 0,10 ; 0,15$; e $0,75 \mathrm{mmol} \mathrm{L}^{-1}$, respectivamente) de cada metal, isolada e simultaneamente $\left(L V_{M I} e\right.$ LVA $_{M I}$ ). As colunas representam as médias e suas respectivas barras indicam o erro-padrão calculado para três repetições. 
Pendias, 2001; Morera et al., 2001). Essa comparação só foi possível porque foram usadas iguais concentrações molares dos metais.

Quando a concentração inicial foi multielementar, composta por $\mathrm{Cd}$, $\mathrm{Cu}$ e $\mathrm{Pb}\left(\mathrm{LV}_{\mathrm{MI}}\right.$ e $\left.\mathrm{LVA}_{\mathrm{MI}}\right)$, houve redução na adsorção dos três metais (Figura 1), sendo esta mais intensa para o $\mathrm{Cd}$. Em estudo de adsorção competitiva com $\mathrm{Cr}, \mathrm{Ni}, \mathrm{Cu}, \mathrm{Zn}, \mathrm{Cd}$ e $\mathrm{Pb}$ foi constatado que quase todos os metais foram adsorvidos pelo solo em concentrações mais baixas, ocupando uma porcentagem proporcional relativa à sua concentração molar na solução; contudo, quando a concentração aumentou, alguns competidores mais fortes, como $\mathrm{Cr}$, $\mathrm{Cu}$ e $\mathrm{Pb}$, mantiveram sua forte afinidade com a superfície adsorvedora, enquanto outros competidores mais fracos, como $\mathrm{Ni}$, $\mathrm{Zn}$ e $\mathrm{Cd}$, foram deslocados, reduzindo, portanto, suas quantidades adsorvidas (Fontes \& Gomes, 2003). Echeverría et al. (1998) observaram que a retenção de $\mathrm{Pb}$ e $\mathrm{Cu}$ foi menos sensível à presença de outros cátions (como $\mathrm{Cd}$, $\mathrm{Ni} \mathrm{e}$ $\mathrm{Zn}$ ), sugerindo uma retenção mais específica, e constataram que o $\mathrm{Cd}$, ao contrário do $\mathrm{Cu}$, influenciou pouco a adsorção de $\mathrm{Pb}$. O Cu e o $\mathrm{Pb}$ têm sido listados como cátions mais competitivos que o $\mathrm{Cd}$ em vários trabalhos (Fontes et al., 2000; Morera et al., 2001).

Segundo McBride (1989, 1994), a retenção de Cd ocorre, na sua maior parte, por meio das forças eletrostáticas das partículas negativamente carregadas, o que a torna altamente dependente da CTC dos solos. Esse comportamento foi constatado também neste trabalho. Por sua vez, as interações de $\mathrm{Cu}$ (Guilherme \& Anderson, 1998) e Pb (Pierangeli et al., 2001) com os colóides dos solos são predominantemente mais específicas e menos dependentes das cargas superficiais. A adsorção específica envolve a troca de metais com ligantes presentes na superfície dos colóides, formando ligações covalentes, e tem sido indicada como causa de alguns solos adsorverem metais acima da sua capacidade prevista com base na CTC (Abd-Elfatah \& Wada, 1981). Agbenin \& Olojo (2004) atribuíram o provável controle da solubilidade de Cu em horizonte B de um
Alfissolo de savana à complexação e, ou, à adsorção específica de $\mathrm{CuOH}^{+}$ou $\mathrm{Cu}^{2+}$ à matéria orgânica e óxidos amorfos. Estudos sugerem um importante papel dos óxidos de Mn em controlar a adsorção de $\mathrm{Pb}$, quando comparado a óxidos de $\mathrm{Fe}$ e material orgânico, ao passo que, para adsorção de $\mathrm{Cd}$, os óxidos de $\mathrm{Fe}$ foram mais importantes que os óxidos de $\mathrm{Al}$ e Mn e material orgânico (Dong et al., 2000). Neste trabalho, os óxidos de $\mathrm{Fe}$ mais reativos $\left(\mathrm{Fe}_{\mathrm{ox}}\right)$, com valores mais elevados no LV (Quadro 1), ajudam a explicar a maior capacidade máxima de adsorção de Cd neste solo (Quadro 2).

$\mathrm{O}$ comportamento diferenciado de $\mathrm{Cd}$ em relação a $\mathrm{Cu}$ e $\mathrm{Pb}$ pode, em parte, ser explicado pela configuração eletrônica desses metais $\left[\mathrm{Pb}^{2+}\right.$ : (Xe) $4 \mathrm{f}^{14} 5 \mathrm{~d}^{10} 6 \mathrm{~s}^{2} ; \mathrm{Cu}^{2+}$ : (Ar) $3 \mathrm{~d}^{9} ; \mathrm{Cd}^{2+}$ : (Kr) $4 \mathrm{~d}^{8} 5 \mathrm{~s}^{2}$ ], em cujas camadas externas $\mathrm{Cu}$ e $\mathrm{Pb}$ possuem elétrons desemparelhados, facilitando as ligações e o compartilhamento de elétrons. Além do mais, os espaços vazios nas orbitais desses dois elementos permitem a formação de complexos, o que não é verificado no $\mathrm{Cd}$.

O coeficiente $\mathrm{K}_{\mathrm{d}}$ (de distribuição), que representa a afinidade de sorção do metal catiônico em solução com a fase sólida do solo, pode ser usado como uma ferramenta para estudos da retenção e mobilidade de metais em solo (Gomes et al., 2001; Mesquita \& Silva, 2002). A seqüência de seletividade de metais em vários solos brasileiros foi estudada por Gomes et al. (2001) e revelou que $\mathrm{Cr}, \mathrm{Pb}$ e $\mathrm{Cu}$ apresentaram valores de $\mathrm{K}_{\mathrm{d}}$ maiores quando comparados com $\mathrm{Cd}$, Zn e Ni. Neste trabalho, os valores de $K_{d}$ (dados não mostrados) corroboram, em termos de tendência geral, os resultados de Gomes et al. (2001), indicando que $\mathrm{Cu}$ e $\mathrm{Pb}$ são mais fortemente retidos quando comparados ao $\mathrm{Cd}$ e que, em estudos de competição, o $\mathrm{Cd}$ pode ser mais facilmente trocado e substituído por $\mathrm{Cu}$ e $\mathrm{Pb}$.

Os solos, de maneira geral, apresentam considerável diversidade de sítios de adsorção, com diferentes graus de afinidade a elementos-traço. Como resultado, a CMAM (Quadro 2) dos solos estudados é mais elevada no $\mathrm{LV}$, para o $\mathrm{Cd}, \mathrm{Cu}$ e $\mathrm{Pb}$, tanto na adsorção individual quanto na simultânea $\left(\mathrm{LV}_{\mathrm{MI}}\right)$. O

Quadro 2. Capacidade máxima de adsorção dos metais Cd, Cu e Pb de Latossolo Vermelho (LV) e Latossolo Vermelho-Amarelo (LVA), calculada pelas equações obtidas da linearização das isotermas de Langmuir

\begin{tabular}{|c|c|c|c|c|c|c|}
\hline \multirow{2}{*}{ Solo } & \multicolumn{2}{|c|}{ Cd } & \multicolumn{2}{|c|}{$\mathrm{Cu}$} & \multicolumn{2}{|c|}{$\mathbf{P b}$} \\
\hline & mmol kg ${ }^{-1}$ & $\mathbf{R}^{2}$ & mmol kg ${ }^{-1}$ & $\mathbf{R}^{2}$ & mmol kg ${ }^{-1}$ & $\mathbf{R}^{2}$ \\
\hline LV & 18,18 & 0,84 & 26,11 & 0,99 & 20,38 & 0,98 \\
\hline LVA & 11,23 & 0,79 & 15,89 & 0,80 & 17,20 & 0,82 \\
\hline $\mathrm{LV}_{\mathrm{MI}}{ }^{(1)}$ & 2,51 & 0,85 & 21,83 & 0,88 & 16,31 & 0,94 \\
\hline $\mathrm{LVA}_{\mathrm{MI}}$ & 0,90 & 0,90 & 18,61 & 0,89 & 10,00 & 0,80 \\
\hline
\end{tabular}

\footnotetext{
${ }^{(1)} \mathrm{MI}$ indica que os metais foram adicionados simultaneamente às amostras de solo.
} 
maior teor de matéria orgânica e a conseqüente maior CTC deste solo em relação ao LVA (Quadro 1) devem estar contribuindo para a sua CMAM ser mais elevada, além de a taxa $\mathrm{Fe}_{\mathrm{ox}} / \mathrm{Fe}_{\mathrm{DCB}}$ ser mais elevada neste solo, indicando presença de mais Fe com menor grau de cristalinidade (Kämpf \& Curi, 2000).

A adsorção simultânea dos três metais revela que há forte efeito competitivo entre eles pelos sítios de adsorção, sendo observadas maiores reduções na CMAM para o $\mathrm{Cd}$, seguido do $\mathrm{Pb}$ e $\mathrm{Cu}$ (Figura $1: \mathrm{LV}_{\mathrm{MI}}$ e $\mathrm{LVA}_{\mathrm{MI}}$ ). A maior redução observada na CMACd indicou que este elemento é retido principalmente nos sítios de troca catiônica, sendo prontamente trocável por elementos com maior afinidade pela matriz do solo, conforme já relatado por Christensen (1989) e Morera et al. (2001). Reduções na CMACu e CMAPb, devido ao efeito competitivo entre elas, também são relatadas por Morera et al. (2001). Fontes et al. (2000), trabalhando com dois Ultissolos e um Oxissolo, relatam que, devido ao efeito competitivo entre $\mathrm{Cd}, \mathrm{Cu}$, $\mathrm{Pb}$ e $\mathrm{Zn}$, há grande redução no teor de $\mathrm{Cd}$ e $\mathrm{Zn}$ adsorvidos por solos tropicais, sendo as isotermas de adsorção melhor ajustadas a um modelo polinomial quadrático. Esses autores verificaram pequena redução na adsorção de $\mathrm{Pb}$ e Cu em decorrência da competição com outros cátions. Entretanto, nos experimentos desses autores as concentrações dos elementos não eram equimolares, o que, de certa forma, dificulta a comparação do comportamento entre os metais.
Tanto na adsorção individual quanto na competitiva, a CMAM segue a seqüência: $\mathrm{Cu}>\mathrm{Pb}>\mathrm{Cd}$, em ambos os solos. Morera et al. (2001) observaram comportamento semelhante em quatro solos da Espanha (três Inceptissolos e um Entissolo) com diferentes propriedades físico-químicas, tanto na adsorção competitiva quanto na individual. Segundo esses autores, esse comportamento indica que esses solos apresentam grupos superficiais com maior afinidade para $\mathrm{Cu}$ e $\mathrm{Pb}$. O menor poder competitivo de $\mathrm{Cd}$, evidenciado pela redução acentuada da CMACd ( $\approx 86 \%$ no LV e $92 \%$ no LVA) na presença dos outros metais, torna esse elemento altamente preocupante em solos tropicais mais intemperizados, como os Latossolos, por indicar maior predisposição desse elemento à movimentação no perfil ou nas águas superficiais.

Em ambos os solos, a dessorção dos três metais foi incompleta, dentro da escala de tempo usada neste experimento: 72 h (Figura 2). Em gibbsita, é relatada reversibilidade total de $\mathrm{Pb}$ em $72 \mathrm{~h}$ (Strawn et al., 1998) e de Cd em 24 h (Weerasooriya et al., 2002). Em solos, a ocorrência de histerese é relatada para $\mathrm{Pb}$ (Strawn \& Sparks, 2000), Cu (Guilherme \& Anderson, 1998) e Cd (McLaren et al., 1998; Tran et al., 2002). Baixa reversibilidade de metais adsorvidos tem sido interpretada como movimento dos íons para o interior de sítios com reações de dessorção mais vagarosas, baixa energia de ativação ou mudanças nas formas químicas dos metais (McBride, 1994;

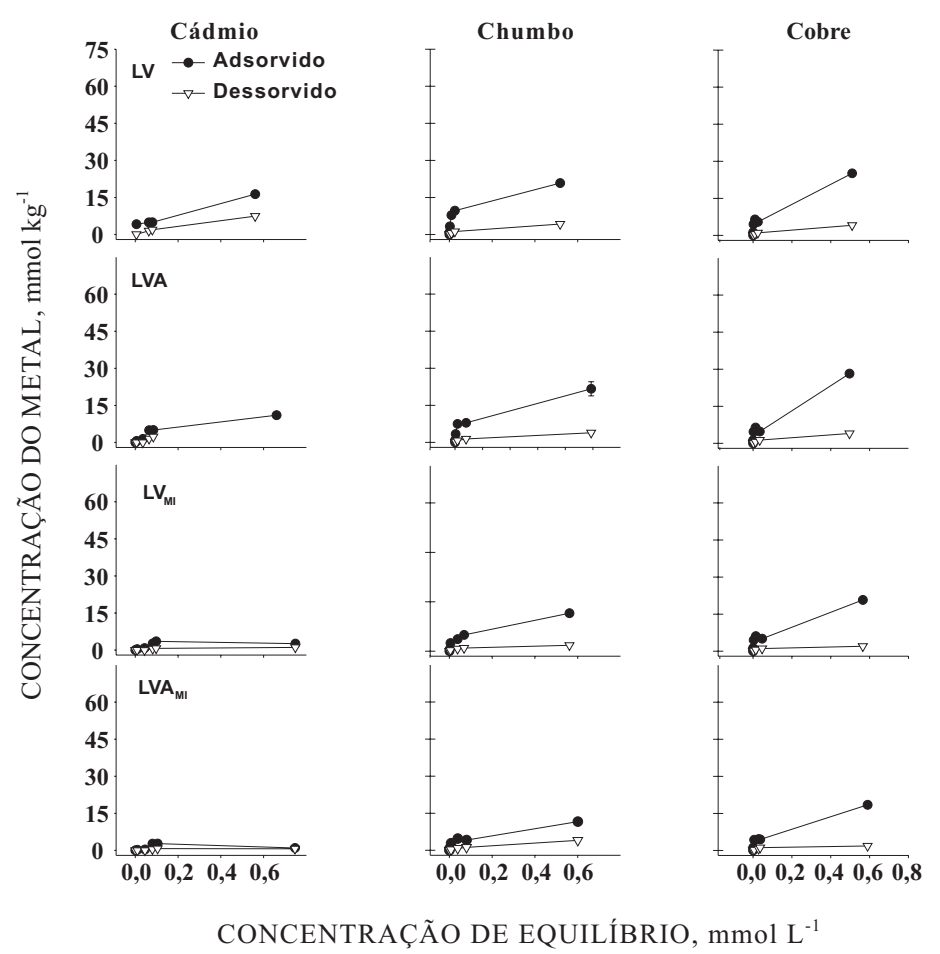

Figura 2. Isotermas de adsorção (๑) e dessorção ( $\nabla)$ dos metais $\mathrm{Cd}$, $\mathrm{Pb}$ e $\mathrm{Cu}$, de amostras de Latossolo Vermelho (LV) e Latossolo Vermelho-Amarelo (LVA), obtidas após reação destas com soluções dos metais, individual e simultaneamente $\left(\mathrm{LV}_{\mathrm{MI}} \mathrm{eLVA}_{\mathrm{MI}}\right)$, nas concentrações de 0,$01 ; 0,05 ; 0,10 ; 0,15 ;$ e $0,75 \mathrm{mmol} \mathrm{L}^{-1}$. 
Backes et al., 1995). A adsorção específica do metal aos colóides do solo também constitui uma explicação adicional para esse fenômeno (Tran et al., 2002).

Tendo como referência as três concentrações iniciais mais elevadas, a porcentagem dessorvida, expressa como a razão entre o metal dessorvido e o metal adsorvido, segue a seqüência: $\mathrm{Cd}>\mathrm{Pb} \approx \mathrm{Cu}$ (Figura 3) na adsorção individual e $\mathrm{Cd}>\mathrm{Pb}>\mathrm{Cu}$ na adsorção simultânea, em ambos os solos. Verifica-se que, além da maior CMAM para $\mathrm{Cu}$ e $\mathrm{Pb}$, esses solos também apresentam menor dessorção desses dois elementos, em relação ao $\mathrm{Cd}$, confirmando maior afinidade para com $\mathrm{Cu}$ e $\mathrm{Pb}$. No entanto, para os três elementos, no tempo estudado, a reversibilidade é baixa, pois, como pode ser visto na figura 3 , menos de $50 \%$ do total de metal adsorvido é dessorvido.

A estimativa do potencial adsorvente do solo, ao lado de informações sobre a reversibilidade das reações de adsorção, fornece subsídios importantes para auxiliar na avaliação do impacto ambiental causado por elementos-traço. No presente estudo, fica evidenciado que a adsorção usando espécies iônicas individuais tem menor aplicação prática para avaliação da retenção de $\mathrm{Cd}$ pelo solo, principalmente quando há presença simultânea de outros metais mais competitivos.

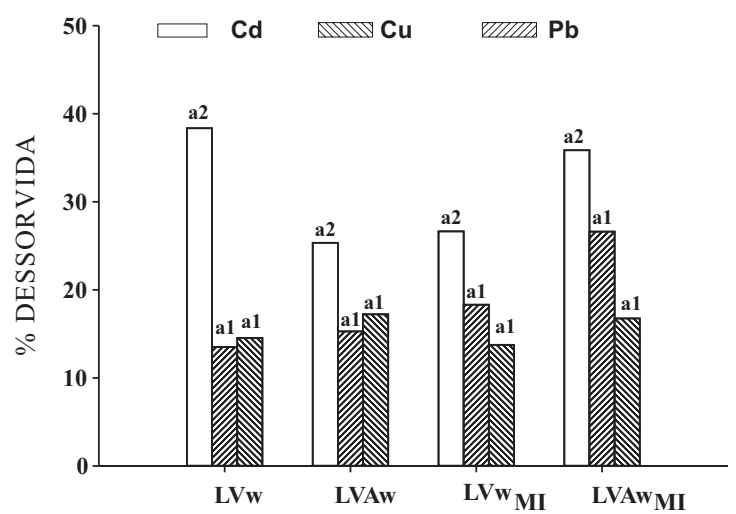

Figura 3. Dessorção porcentual de metal (M) $\left.\left[\mathbf{M}_{\text {dessorvido }} / \mathbf{M}_{\text {adsorvido }}\right) \times 100\right]$ de amostras de Latossolo Vermelho (LV) e Latossolo VermelhoAmarelo (LVA) em solução de $\mathrm{Ca}\left(\mathrm{NO}_{3}\right)_{2}$ $5 \mathrm{mmol} \mathrm{L}^{-1}$, após reação das mesmas com soluções dos metais, individualmente e simultaneamente (MI), nas concentrações de 0,10; 0,15; e 0,75 $\mathrm{mmol} \mathrm{L}^{-1}$. Barras seguidas de mesma letra, dentro do mesmo solo, não diferem entre si pelo teste de Scott-Knott a $5 \%$.

\section{CONCLUSÕES}

1. Em ambos os solos, a adsorção de $\mathrm{Cd}$ foi consistentemente menor que a de $\mathrm{Cu}$ e $\mathrm{Pb}$, ocorrendo tendência inversa na dessorção.

2. A adsorção simultânea reduziu a retenção dos três metais, sendo a maior redução verificada para o $\mathrm{Cd}$.
3. O menor poder competitivo de $\mathrm{Cd}$ indica sua maior predisposição à movimentação no perfil de Latossolos ou nas águas superficiais.

4. Os atributos mineralógicos mostraram-se menos importantes que os químicos nestes solos altamente intemperizados, no tocante à retenção de $\mathrm{Cd}, \mathrm{Cu}$ e Pb.

\section{AGRADECIMENTOS}

Ao Professor Ruy Carvalho, do Departamento de Química da UFLA, e à Pesquisadora do IAC, Cleide Aparecida de Abreu, pelas críticas e sugestões.

\section{LITERATURA CITADA}

ABD-ELFATTAH, A. \& WADA, K. Adsorption of lead, copper, zinc, cobalt, and cadmium by soils that differ in cationexchange materials. J. Soil Sci., 32:271-283, 1981.

ABRAHAMS, P.W. Soils: Implications to human health. Sci. Total Environ., 291:1-32, 2002.

AGBENIN, J.O. \& OLOJO, L.A. Competitive adsorption of copper and zinc by a Bt horizon of a savana Alfisol as affected by $\mathrm{pH}$ and selective removal of hydrous oxides and organic matter. Geoderma., 119:85-95, 2004.

ALTIN, O.; ÖZBELGE, H.Ö. \& DOGU, T. Use of general purpose adsorption isotherms for heavy metal-clay mineral interactions. J. Colloid Interface Sci., 130:130140,1998

AMARAL SOBRINHO, N.M.B.; COSTA, L.M.; OLIVEIRA, C. \& VELLOSO, A.C.X. Metais pesados em alguns fertilizantes e corretivos. R. Bras. Ci. Solo, 16:271-276, 1992.

BACKES, C.A.; McLAREN, R.G.; RATE, A.W. \& SWIFT, R.S. Kinetics of cadmium and cobalt desorption from iron and manganese oxides. Soil Sci. Soc. Am., J.59:778-785, 1995.

BRADL, H.B. Adsorption of heavy metal ions on soils and soils constituents. J. Colloid Interface Sci., 277:1-18, 2004.

CAMPBELL, L.S. \& DAVIES, B.E. Soil sorption of cesium modelled by the Langmuir and Freundlich isotherms equations. Appl. Geochem., 10:715-723, 1995.

CHRISTENSEN, T.H. Cadmium soil sorption at low concentrations. V. Evidence of competition by other heavy metals. Water Air Soil Poll., 34:293-303, 1989.

DONG, D.; NELSON, Y.M.; LION, L.W.; SHULER, M.L. \& GHIORSE, W.C. Adsorption of $\mathrm{Pb}$ and $\mathrm{Cd}$ onto metal oxides and organic material in natural surface coatings as determined by selective extractions: New evidence for the importance of Mn and Fe oxides. Water Res., 34:427436, 2000. 
ECHEVERRÍA, J.C.; MORERA, M.T.; MAZKIARÁN, C. \& GARRIDO, J.J. Competitive sorption of heavy metal by soils. Isotherms and fractional factorial experiments. Environ. Poll., 101:275-284, 1998.

FONTES, M.P.F. \& GOMES, P.C. Simultaneous competitive adsorption of heavy metals by the mineral matrix of tropical soils. Appl. Geochem., 18:795-804, 2003.

FONTES, M.P.F.; MATOS, A.T.; COSTA, L.M. \& NEVES, J.C.L. Competitive adsorption of zinc, cadmium, copper, and lead in three highly-weathered Brazilian soils. Comm. Soil Sci. Plant Anal., 31:2940-958, 2000.

GOMES, P.C.; FONTES, M.P.F.; SILVA, A.G.; MENDONÇA, E.S. \& NETTO, A.R. Selectivy sequence and competitive adsorption of heavy metals by Brazilian soils. Soil Sci Soc. Am. J., 65:1115-1121, 2001.

GUILHERME, L.R.G. \& ANDERSON, S.J. Copper sorption kinetics and sorption hysteresis in two oxide-rich soils (Oxisols): Effect of phosphate pretreatment. In: JENNE, E.A., ed. Adsorption of metals by geomedia: Variables, mechanisms, and model applications. San Diego, Academic Press, 1998. p.209-228.

KABATA-PENDIAS, A. \& PENDIAS, H. Trace elements in soils and plants. 3.ed. Boca Raton, CRC Press, 2001. 413p.

KÄMPF, N. \& CURI, N. Óxidos de ferro: Indicadores de ambientes pedogênicos e geoquímicos. In: NOVAIS, R.F.; ALVAREZ V., V.H. \& SCHAEFER, C.E.G.R., eds. Tópicos em ciência do solo. Viçosa, MG, Sociedade Brasileira de Ciência do Solo, 2000. v.1. p.107-138.

LEHMANN, R.G. \& HARTER, R.D. Assessment of copper-soil bond strength by desorption kinetics. Soil Sci. Soc. Am. J., 48:769-772, 1984.

LIMA, G.J.E.O. Biodisponibilidade de cádmio, zinco e chumbo presentes em calcário comercial para o feijoeiro cultivado em três solos do Noroeste de Minas Gerais. Lavras, Universidade Federal de Lavras 2001. 148p. (Tese de Mestrado)

LIMA, J.M. \& ANDERSON, S.J. Effect of aggregation and aggregate size on extractable $\mathrm{Fe}$ and $\mathrm{Al}$ in two Brazilian Typic Hapludoxs. Soil Sci. Soc. Am., 61:965-970, 1997.

McBRIDE, M.B. Environmental chemistry of soils. New York, Oxford University Press, 1994. 406p.

McBRIDE, M.B. Reactions controlling heavy metals in soils. Adv. Soil Sci., 10:1-56, 1989.

McLAREN, R.G.; BACKES, C.A.; TATE, A.W. \& SWIFT, R.S. Cadmium and cobalt desorption kinetics from soil clays: Effect of sorption period. Soil Sci. Soc. Am., 62:332-337, 1998.
MESQUITA, M.E. \& SILVA, J.M.V. Preliminary study of $\mathrm{pH}$ effect in the application of Langmuir and Freundlich isotherms to $\mathrm{Cu}-\mathrm{Zn}$ competitive adsorption. Geoderma, 116:219-234, 2002.

MORERA, M.T.; ECHEVERRIA, J.C.; MAZKIARÁN, C. \& GARRIDO, J.J. Isotherms and sequential extraction procedures for evaluating sorption and distribution of heavy metals in soils. Environ. Poll., 113:135-144, 2001.

PIERANGELI, M.A.P.; GUILHERME, L.R.G.; OLIVEIRA, L.R.; CURI, N. \& SILVA, M.L.N. Efeito da força iônica da solução de equilíbrio sobre a adsorção/dessorção de chumbo em Latossolos brasileiros. Pesq. Agropec. Bras., 36:1077-1084, 2001.

POMBO, L.C.A. Sorção de cádmio em solos do Estado do Rio Grande do Sul. R. Bras. Ci. Solo, 19:19-24, 1995.

RAMALHO, J.F.G.P.; AMARAL SOBRINHO, N.M.B. \& VELLOSO, A.X. Contaminação da microbacia de Caetés com metais pesados pelo uso de agroquímicos. Pesq. Agropec. Bras., 35:1289-1303, 2000.

RIBEIRO-FILHO, M.R.; CURI, N.; SIQUEIRA, J.O. \& MOTTA, P.E.F. Metais pesados em solos de áreas de rejeitos de indústria de processamento de zinco. R. Bras. Ci. Solo, 23:453-464, 1999 .

SILVA, M.L.N.; CURI, N.; MARQUES, J.J.G.; GUILHERME, L.R.G. \& LIMA, J.M. Ponto de efeito salino nulo e suas relações com propriedades mineralógicas e químicas de Latossolos brasileiros. Pesq. Agropec. Bras., 31:663-671, 1996.

SPARKS, D.L. Environmental soil chemistry. San Diego, Academic Press, 1995. 237p.

STRAWN, D. \& SPARKS, D.L. Effects of soil organic matter on the kinetics and mechanisms of $\mathrm{Pb}$ (II) sorption and desorption in soil. Soil Sci. Soc. Am., 64:144-156, 2000.

STRAWN, G.D.; SCHEIDEGGER, A.M. \& SPARKS, D.L. Kinetics and mechanisms of $\mathrm{Pb}(\mathrm{II})$ sorption and desorption at the aluminum oxide-water interface. Environ. Sci. Technol., 32:2596-2601, 1998.

TEMMINGHOFF, E.J.M.; van der ZEE, S. \& DE HAAN, F.A.M. Speciation and calcium competition effects on cadmium sorption by sandy soil at various pHs. Eur. J. Soil Sci., 46:649-655, 1995

TRAN, Y.T.; BARRY, D.A. \& BAJRACARYA, K. Cadmium desorption in sand. Environ. Intern., 28:493-502, 2002.

VEITH, J.A. \& SPOSITO, G. On the use of the Langmuir equation in the interpretation of "adsorption" phenomena. Soil Sci. Soc. Am. J., 41:697-702, 1977.

WEERASOORIYA， R.; WIJESEKARA， H.K.D.K. \& BANDARA, A. Surface complexation modeling of cadmium adsorption on gibbsite. Colloids Surfaces A: Physicochem. Eng. Aspects, 207:13-24, 2002. 